\title{
Zero and first-order phase shift correction for field map estimation with dual-echo GRE using bipolar gradients ${ }^{\text {tर }}$
}

\author{
Desmond T.B. Yeo ${ }^{\mathrm{a}, \mathrm{b}}$, Thomas L. Chenevert ${ }^{\mathrm{a}}$, Jeffrey A. Fessler ${ }^{\mathrm{a}, \mathrm{b}}$, Boklye Kim ${ }^{\mathrm{a}, *}$ \\ ${ }^{a}$ Department of Radiology, University of Michigan Medical School, Ann Arbor, MI 48109, USA \\ ${ }^{\mathrm{b}}$ Department of Electrical Engineering and Computer Science, University of Michigan, Ann Arbor, MI 48109, USA \\ Received 27 October 2006; revised 9 February 2007; accepted 10 February 2007
}

\begin{abstract}
A simple phase error correction technique used for field map estimation with a generally available dual-echo gradient-echo (GRE) sequence is presented. Magnetic field inhomogeneity maps estimated using two separate GRE volume acquisitions at different echo times are prone to dynamic motion errors between acquisitions. By using the dual-echo sequence, the data are collected during two back-to-back readout gradients in opposite polarity after a single radio frequency pulse, and interecho motion artifacts and alignment errors in field map estimation can be factored out. Residual phase error from the asymmetric readout pulses is modeled as an affine term in the readout direction. Results from phantom and human data suggest that the first-order phase correction term stays constant over time and, hence, can be applied to different data acquired with the same protocol over time. The zero-order phase correction term may change with time and is estimated empirically for different scans.
\end{abstract}

(C) 2007 Elsevier Inc. All rights reserved.

Keywords: Field-inhomogeneity; Field map; Dual-echo; Phase correction

\section{Introduction}

The static magnetic field passing through an object in a magnetic resonance imaging (MRI) scanner is perturbed by disjoint object regions with different magnetic susceptibilities that augment the magnetic field inhomogeneity caused by imperfections in the gradients and main magnet. Field inhomogeneity causes image artifacts that increase in severity as the static magnetic field strength, $B_{0}$, increases. Such artifacts are especially apparent in high-speed MRI techniques like echo-planar imaging and spiral imaging, where geometric distortion and blurring are observed, respectively, because of the longer readout time. Most correction methods for field inhomogeneity effects require an accurate estimate of the field map $[1,2]$. These methods assume that the data from

\footnotetext{
This work was supported in part by the National Institute of Health grant 1P01 CA87634 \& R01 EB00309.

* Corresponding author. Basic Radiological Sciences Division, Department of Radiology, University of Michigan Medical School, MI 48109-2200, USA. Tel.: +1 734763 5692; fax: +1 7346151471 .

E-mail address: boklyek@umich.edu (B. Kim).
}

two different echo times acquired for field map estimation are free of acquisition dependant errors, i.e., position changes due to motion.

A static field map can be estimated by taking the phase difference of a pair of gradient-echo (GRE) images acquired at two different echo times [3-5]. The echo time difference is typically constrained to be small to prevent phase wrapping. With a few exceptions, field maps are generated using two separate image acquisitions with different echo times. However, this method is prone to motion-induced and position-dependent errors that degrade the field map. Using two separate radio frequency (RF) excitations with different echo times would produce accurate field maps only in the absence of motion, i.e., phantom studies. Ideally, $B_{0}$ field maps may be computed from the phase changes between two time points of the same images. In human data sets, a common problem of computing field maps from two different images, acquired at two different echo times, is the change in $B_{0}$ during the time delay due to the motion, either bulk head motion or physiological brain motion, which causes the error in field map measurement. Typical acquisition times for 3D spoiled GRASS (SPGR) volumes used for the field map computations are approximately 3-4 min. With a normal subject, 
A

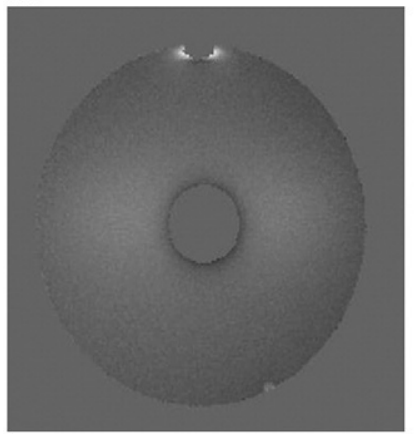

B

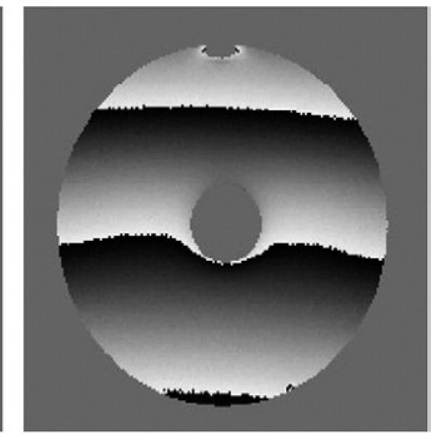

Fig. 1. Off-resonance maps of phantom estimated by standard off-resonance method (A) and uncorrected dual-echo method showing linear phase wrapping in readout direction ( $x$ direction downwards) (B).

the mean translation and rotation of the head were observed to be $2.25 \mathrm{~mm}$ and $0.71^{\circ}$, respectively, in a 3-min scan time [6]. Even if the head is restrained, brain tissue velocity for normal subject could be $0.94 \pm 0.26 \mathrm{~mm} / \mathrm{s}$ due to the physiological motion [7]. The corresponding images from the two separate volumes with typical 3-min acquisition time will then be misregistered, resulting in the field map estimation error. There is a clear advantage in measuring a field map from the same images acquired at two different echo times, i.e., using a dual-echo sequence.

Partial $k$-space techniques for dynamic field map estimation can greatly reduce motion-induced errors but may suffer from decreased field map resolution [8]. Some echo planar imaging-based dynamic field map estimation methods acquire the field maps in distorted space, obviating the need for registration between the field maps and the geometrically distorted echo planar images [8,9]. Other field inhomogeneity correction methods assume that the field map is available in undistorted space $[10,11]$. In some dynamic field mapping techniques, dual-echo images are acquired by using the same positive polarity in the read out gradient, but that would require pulse sequence modifications, an option that may not be available on all clinical scanners.

This work presents a zero- and first-order phase shift correction technique used in conjunction with a simple dualecho fast GRE (DEFGRE) pulse sequence employing two back-to-back readout gradients, continuous but of opposite polarity, for static field map estimation. This work describes a relatively straightforward technique that allows computation of field maps without the need to modify a commonly available sequence in a clinical setup where the sequence modification is not accessible. The pulse sequence, DEFGRE, acquires two echoes efficiently with one RF pulse, and the image data can be used to compute field maps without motion-induced position errors. A caveat in using this sequence is that, due to the asymmetry of the readout gradients, artifactual phase shifts causing phase wraps are evident in the phase difference map. This study focuses on correcting this residual phase error to remove the phase wraps without using elaborate phase unwrapping algorithms $[12,13]$. We formulate a hypothesis of how the asymmetric readout pulses cause the artifactual phase shift and then model the phase error as an affine term in the readout direction. The unknown affine model parameters are then estimated using motionless phantom data. Results from several sets of phantom and patient data acquired on the same scanner with the same scan parameters over a period of 2 years suggest that the first-order phase correction term does not change for a given scanner over time and, hence, can be applied to the field map estimation of different data sets. The zero-order phase correction term may change with time but can be estimated empirically from the dual-echo data for each new scan.

\section{Methods}

\subsection{DEFGRE pulse sequence}

In the generally used static field map estimation method, two complex images, $I_{\mathrm{TE} 1 \text {,sep }}$ and $I_{\mathrm{TE} 2 \text {,sep}}$, are acquired

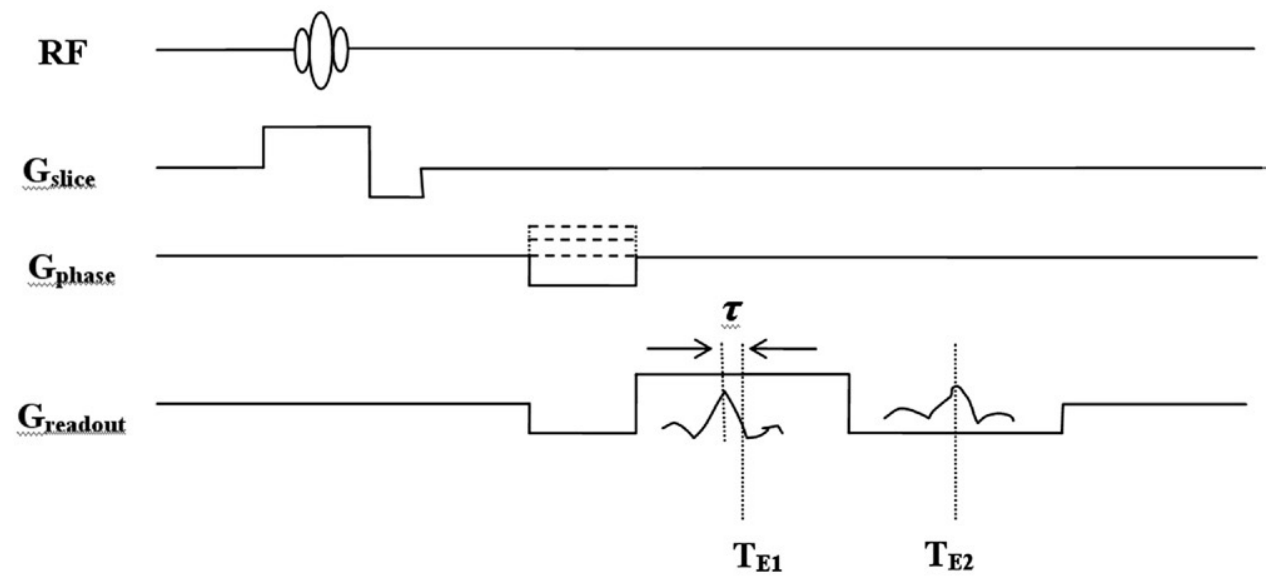

Fig. 2. Simplified dual-echo pulse sequence with back-to-back $G_{\text {readout }}$ pulses with opposite polarity. Readout data from $\mathrm{T}_{\mathrm{E} 1}$ may be off-center relative to data from $\mathrm{T}_{\mathrm{E} 2}$. The first-order phase shift correction term $\alpha$ is proportional to the time delay $\tau$. 


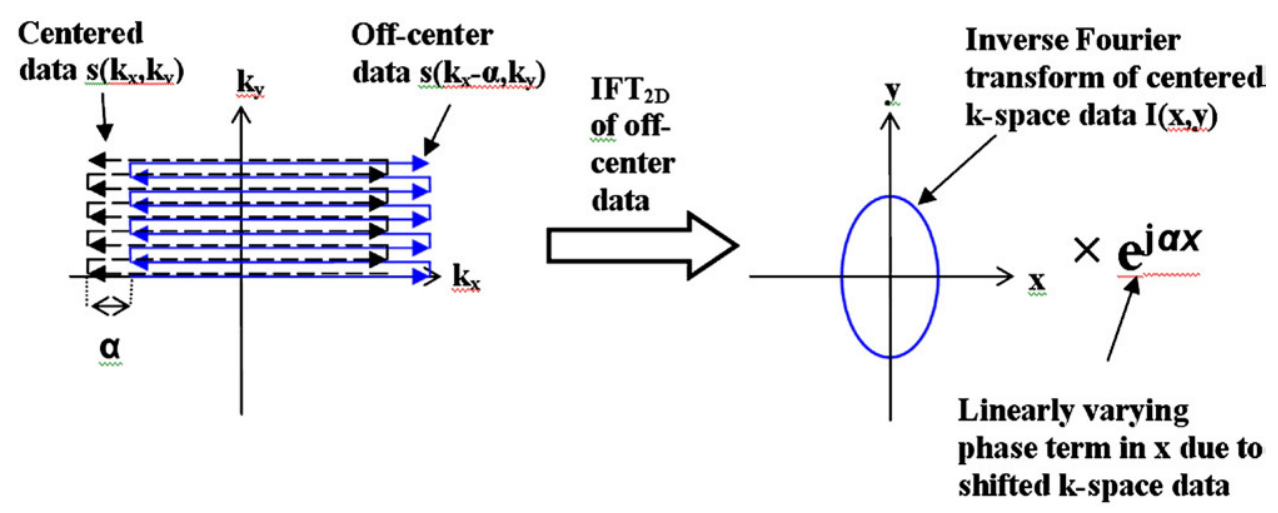

Fig. 3. Frequency shifted $k$-space data is transformed via inverse Fourier transform to an image with a first-order phase shift term in the readout direction $x$.

separately at two different echo times, TE1 and TE2, where TE2 $>$ TE1. Assuming all scan parameters, excluding the echo time, are identical for the two sets of images, $I_{\mathrm{TE} 2 \text {,sep }}$ will approximately be equal to the complex magnetization $M_{\text {sep }}$ of $I_{\mathrm{TE} 1 \text {,sep }}$, multiplied by a complex phase term dependent on the field inhomogeneity. The two sets of images can be written as

$I_{\mathrm{TE} 1, \mathrm{sep}}(r)=M_{\mathrm{sep}}(r)+\varepsilon_{1}(r)$,

$I_{\mathrm{TE} 2 \text {,sep }}(r)=M_{\mathrm{sep}}(r) e^{j\left(\Delta \omega_{\mathrm{sep}}(r) \Delta T E_{\mathrm{sep}}\right)}+\varepsilon_{2}(r)$

where the spatial variable is $r=\left[\begin{array}{lll}x & y & z\end{array}\right]^{T}$, the true offresonance map is denoted by $\Delta \omega_{\text {sep }}, \Delta \mathrm{TE}=\mathrm{TE} 2-\mathrm{TE} 1$ and the images have complex noise denoted by $\varepsilon_{1}$ and $\varepsilon_{2}$. The off-resonance map can be estimated as

$\Delta \hat{\omega}_{\mathrm{sep}}(r)=\frac{\angle\left[I_{\mathrm{TE} 2 \text {,sep }}(r) I_{\mathrm{TE} 1, \mathrm{sep}}^{*}(r)\right]}{\Delta T E_{\mathrm{sep}}} \approx \Delta \omega_{\mathrm{sep}}(r)$,

where $I_{\mathrm{TE} 1 \text {,sep }}^{*}$ denotes the complex conjugate of $I_{\mathrm{TE} 1 \text {,sep }}$. Fig. 1A shows an example of an off-resonance map estimated with Eq. (3).

In the proposed dual-echo field map method, two complex images $I_{\mathrm{TE1} \text {,dual }}$ and $I_{\mathrm{TE2} \text {,dual }}$ are acquired with back-to-back readout gradients. There is no delay between the pulses, which have opposite polarity, as shown in Fig. 2. Due to imperfect gradient balancing along the readout direction, as in most scanners, the two sampled echoes for each readout line may not be centered relative to each other in the readout direction in $k$-space. Assuming that the gradient imbalance is relatively constant for every scan, we model this nonideal behavior as a net shift of one of the $k$-space echo data relative to the other in the readout direction, as shown in Fig. 3. This frequency shift induces a spatially linear, first-order phase shift term, $e^{j \alpha x}$, in the readout direction in the image domain. This term would cause massive phase wrapping in the readout direction if the general field map estimation procedure in Eq. (3) were applied to the dual-echo data under the unrealistic assumption that the gradient pulses are symmetric.
Ignoring $\mathrm{T}^{2}$ relaxation effects, we model $I_{\mathrm{TE2} \text {,dual }}$ as the complex magnetization of $I_{\mathrm{TE} 1 \text {,dual, }}$, denoted by $M_{\text {dual }}$, multiplied by several complex terms, as follows:

$I_{\mathrm{TE} 1, \text { dual }}(r)=M_{\text {dual }}(r)+\varepsilon_{3}(r)$

$I_{\mathrm{TE} 2 \text {,dual }}(r)=M_{\text {dual }}(r) e^{j\left(\Delta \omega_{\mathrm{sep}}(r) \Delta T E_{\text {dual }}\right)} e^{j(\alpha x+\beta)}+\varepsilon_{4}(r)$

where the field inhomogeneity-induced complex term is denoted by $e^{j\left(\Delta \omega_{\text {sep }}(\mathbf{r}) \Delta T E_{\text {dual }}\right)}$ and the first-order phase shift is modeled by $e^{j \alpha x}$, where $x$ is the readout direction. The complex term $e^{j \beta}$ attempts to model any residual zero-order phase shift left over after the first-order phase component has been removed. Multiplying Eq. (4) by the complex conjugate of Eq. (5) and dividing by its magnitude, we obtain

$$
\begin{aligned}
I_{\mathrm{dual}}(r) & =I_{\mathrm{TE} 2 \text {,dual }}(r) I_{\mathrm{TE} 1, \text { dual }}^{*}(r) /\left|I_{\mathrm{TE} 1 \text {,dual }}^{*}(r)\right| \\
& =\left|M_{\text {dual }}(r)\right| e^{j\left(\Delta \omega_{\text {sep }}(r) \Delta T E_{\text {dual }}\right)} e^{j(\alpha x+\beta)}+\varepsilon_{5}(r)
\end{aligned}
$$

where the phase of the complex magnetization $M_{\text {dual }}$ cancels out. The off-resonance map can be estimated by taking the ratio of the phase of $I_{\text {dual }}$ and $\Delta \mathrm{TE}_{\text {dual }}$,

$\Delta \hat{\omega}_{\text {dual }}(r)=\frac{\angle\left[I_{\text {dual }}(r)\right]}{\Delta T E_{\text {dual }}}=\Delta \omega_{\text {sep }}(r)=+\frac{\alpha x+\beta}{\Delta T E_{\text {dual }}}+\eta_{1}(r)$.

However, for the DEFGRE acquisitions, this estimate of the field map is highly inaccurate unless the massive phase wrapping caused by the first-order phase term $e^{j \alpha x}$, as shown in Fig. 1B, is removed.

\subsection{Residual phase error correction}

To obtain field maps with DEFGRE, we assume that the affine phase parameters $\alpha$ and $\beta$ are independent of the object being scanned, in which case, they need to be calibrated only once for all the data acquired in the same scanner with a given set of imaging parameters, i.e., imaging sequence and field of view. A phantom filled with doped water, which has a well-defined homogeneous region, was scanned for the purpose of computing the 
calibration term: first, using 2D fast SPGR sequence; twice, at different echo times followed by a dual-echo acquisition using DEFGRE sequence. The off-resonance map estimates $\Delta \hat{\omega}_{\text {sep }}$ and $\Delta \hat{\omega}_{\text {dual }}$ were then computed using Eqs. (3) and (7), respectively. Since the spherical phantom is motionless, it is reasonable to consider $\Delta \hat{\omega}_{\text {sep }}$ to be the ground truth of the phantom field map. We estimate the correction parameters $\alpha$ and $\beta$ by minimizing the following cost function with phantom data:

$$
\begin{aligned}
\hat{\alpha}, \hat{\beta}= & \underset{\alpha, \beta}{\arg \min } \sum_{x=0}^{N-1} \mid \angle\left\{\exp \left[j \frac{\alpha x+\beta-\Delta \hat{\phi}_{\text {dual }}\left(x, y_{0}\right)}{\Delta T E_{\text {dual }}}\right]\right\} \\
& -\left.\angle\left\{\exp \left(j \Delta \hat{\omega}_{\text {sep }}\left(x, y_{0}\right)\right)\right\}\right|^{2},
\end{aligned}
$$

where $y_{0}$ is a column of $N$ pixels for which $\left|M_{\text {dual }}\right|$ is significantly large, i.e., $\left|M_{\text {dual }}\right|$ exceeds $10 \%$ of the maximum image intensity of dual echo data, $\Delta \hat{\phi}_{\text {dual }}$ is the estimate of the dual-echo phase difference map or $\angle\left[I_{\text {dual }}\right], \Delta \mathrm{TE}_{\text {dual }}$ is the time difference between the two echoes in the DEFGRE sequence, $y$ is the phase encoding direction, $x$ is the readout direction and $\Delta \hat{\omega}_{\text {sep }}$ is the estimate of the off-resonance map obtained with Eq. (3). The first-order phase error $\alpha$ is solely dependent on the gradient imbalance and should not change considerably with different $\Delta \mathrm{TE}_{\text {dual }}$. The estimation of $\alpha$ will serve to unwrap the linear component of the phase error. Data from a single column $y_{0}$ is used in Eq. (8) since the firstorder phase shift is modeled in the readout or $x$ direction. The values of $\alpha$ and $\beta$ estimated via the Nelder-Mead simplex method are used to correct the dual-echo field map acquired for subsequent studies. All phase correction computational work was performed on an Intel Pentium 4 3.6 GHz CPU using MATLAB (The Mathworks, Natick, MA, USA).

\subsection{Empirical approximation of $\beta$}

Table 1 reports that while $\alpha$ remained constant over different scans of phantoms on the same scanner, $\beta$ varied with different scan sessions. This indicates that $\alpha$ has to be estimated only once for a given scanner. As the goal of this study is to be able to compute a field map from the DEFGRE data directly without the need to acquire additional data for human studies, an empirical method to estimate $\beta$ was implemented. As the study progressed, $\beta$ was determined for a new human subject scan by computing the difference between the mean of two single-echo SPGR off-resonance values, over several homogeneous regions in several previously scanned images from different human subjects and the corresponding mean DEFGRE off-resonance value of the new subject after linear phase correction with $\alpha$. This difference is computed in hertz, and $\beta$ is then estimated by multiplying the off-resonance difference by $2 \pi \Delta \mathrm{TE}_{\text {dual }}$. The two single-echo SPGR off-resonance values of the homogeneous regions across the scanned subjects used to calculate the mean value show little variation (S.D. of $2.22 \mathrm{~Hz}$ ).

\subsection{Phantom and human subject data}

Along with the homogeneous sphere phantom $(17 \mathrm{~cm}$ in diameter) filled with Gadolinium-doped water, an air-water phantom (i.e., susceptibility phantom) representing susceptibility changes in a typical human head was imaged. The susceptibility phantom was constructed with a cylinder (13 $\mathrm{cm}$ in diameter and $20 \mathrm{~cm}$ in height) filled with doped water and a lateral air column suspended in the middle, which induces inhomogeneity in the static magnetic field. Each phantom was scanned with two pulse sequence protocols: (i) 2D DEFGRE (with readout gradients in opposite polarities $)(\mathrm{TR}=200 \mathrm{~ms}, \mathrm{TE} 1=2.6 \mathrm{~ms}$, TE2 $=$ $5.3 \mathrm{~ms}, \Delta \mathrm{TE}=2.7 \mathrm{~ms}$, image matrix $=256 \times 256 \times 68)$ and (ii) twice with single-echo $2 \mathrm{D}$ SPGR $(\mathrm{TR}=200 \mathrm{~ms}$, image matrix $=256 \times 256 \times 68)$ at $\mathrm{TE} 1=2.7 \mathrm{~ms}$ and TE2 $=4.2 \mathrm{~ms}$, where $\Delta \mathrm{TE}=1.5 \mathrm{~ms}$. The slice locations were kept consistent with the dual-echo data. All the above scans were performed twice on each phantom in an interval of 4 months on the same 1.5 T GE SIGNA MR scanner (GE Medical Systems, Milwaukee, WI, USA).

In addition, data from three different human subjects were acquired over a period of two years after the first phantom scan. The studies were conducted in accordance with the guidelines set by the University of Michigan Medical School Internal Review Board. Informed consent was obtained from all three subjects. The data were acquired with two pulse sequence protocols: (i) 2D DEFGRE $(\mathrm{TE} 1=2.7 \mathrm{~ms}, \mathrm{TE} 2=5.3 \mathrm{~ms}, \Delta \mathrm{TE}=2.6 \mathrm{~ms}$, image matrix $=256 \times 256 \times 54$ ) and (ii) two single-echo $3 \mathrm{D}$ SPGR $(\mathrm{TE} 1=2.4 \mathrm{~ms}, \mathrm{TE} 2=4.2 \mathrm{~ms}, \Delta \mathrm{TE}=1.8 \mathrm{~ms}$, image matrix $=256 \times 256 \times 128$ ). All phantom and human scans were performed on the same scanner.

The first-order phase correction terms are useful if they are constant over time for a given scanner and can be applied to yield corrected field maps from dual-echo acquisitions. For phantom data, the corrected field map can be validated with the truth map, i.e., a field map

Table 1

Estimated phase correction parameters for phantom data acquired on same scanner using (i) DEFGRE and 2D SPGR data with Eq. (8) (first two rows) and (ii) DEFGRE data and mean 2D SPGR off-resonance value with empirical method

\begin{tabular}{llcr}
\hline & \multicolumn{2}{c}{ Estimated parameters for phantom data } & Scan 2 (4 months later) (sphere) \\
\cline { 2 - 4 } & Scan 1 (susceptibility) & Scan 2 (4 months later) (susceptibility) & -0.10 \\
$\beta$ (rad/unit distance) & -0.10 & -0.10 & 0.11 \\
$\beta(\mathrm{rad})$ using Eq. (8) & 2.26 & 0.27 & 0.12 \\
\hline
\end{tabular}




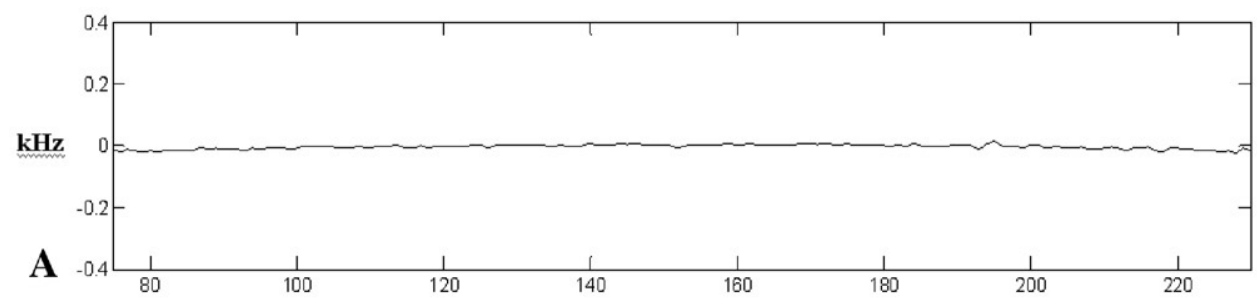

n

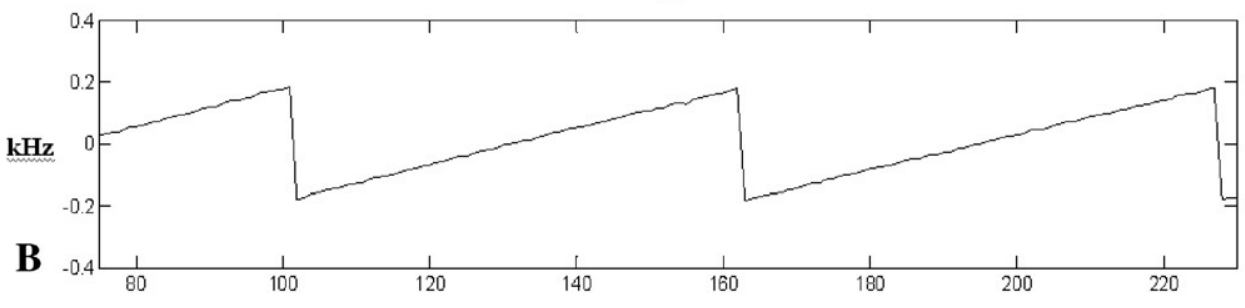

n

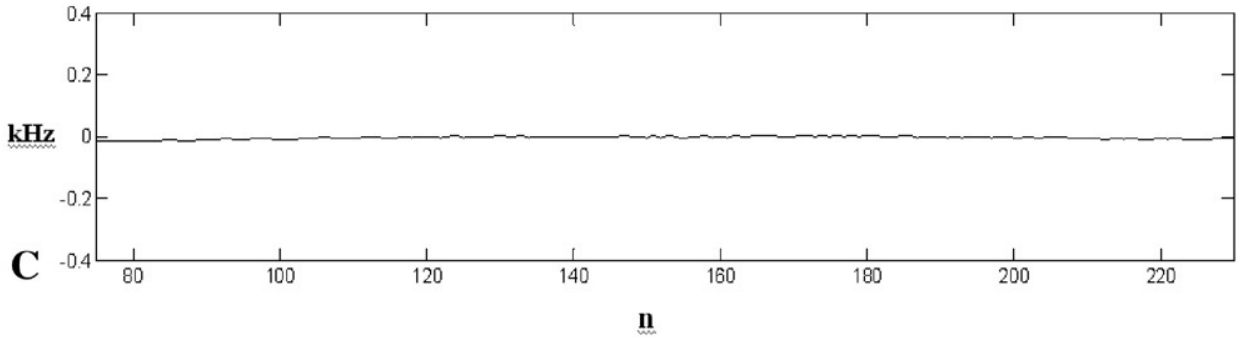

Fig. 4. A column of the spherical phantom off-resonance map samples in the readout direction for standard off-resonance method (A), dual-echo off-resonance method (B) and corrected dual-echo off-resonance method (C).

calculated from two separate single echo acquisitions. The constant first-order phase correction terms can then be routinely applied to calculate the initial field map from a dual-echo acquisition for the correction of the $B_{0}$ inhomogeneity that induces image distortions in clinical human data. The values from the homogeneous phantom regions
A
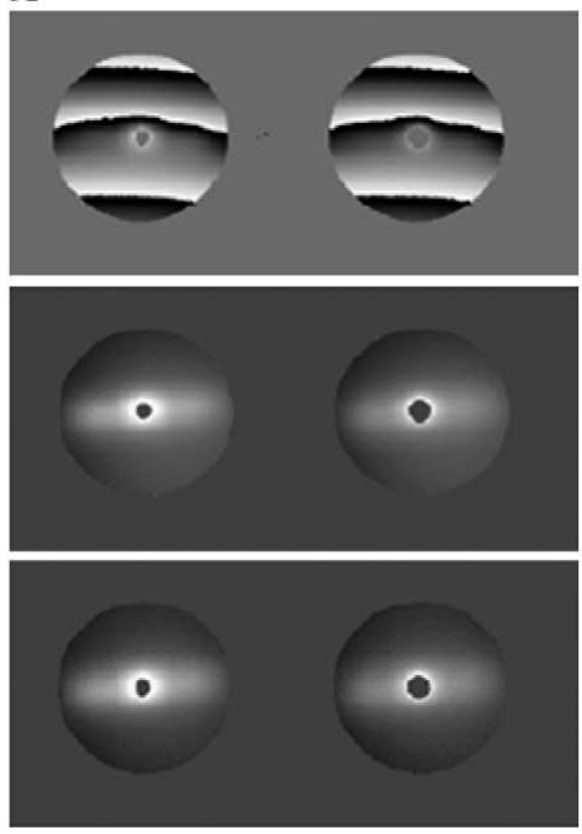

\section{B}
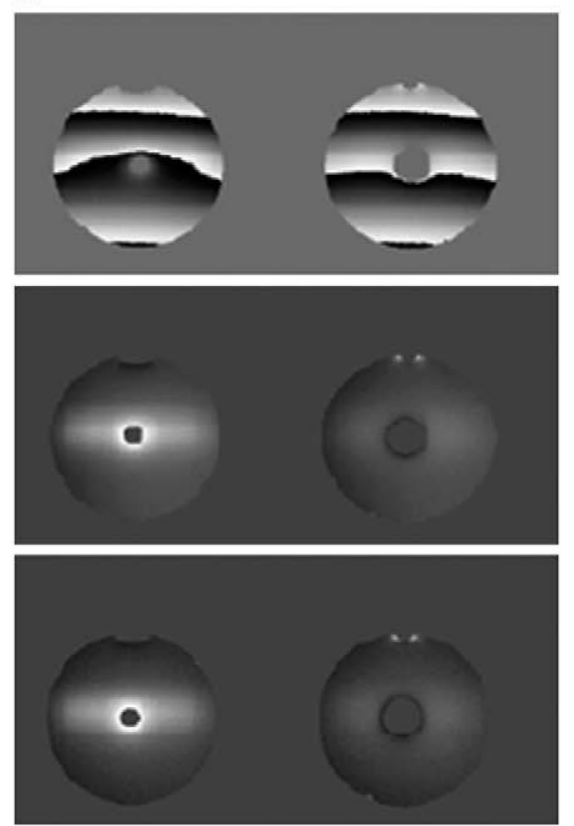

C
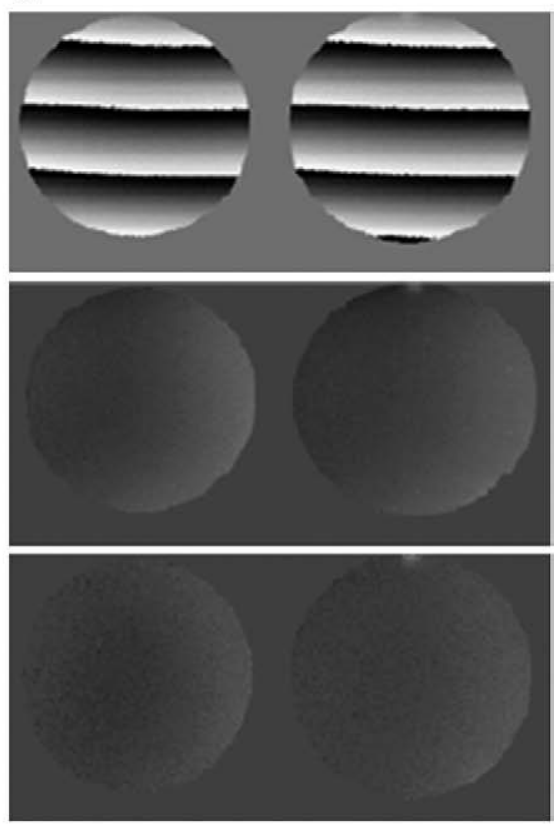

Fig. 5. Two slices of off-resonance maps in hertz from DEFGRE without correction (top), DEFGRE after correction with affine phase term (middle) and two separate single-echo acquisitions (bottom) for susceptibility phantom in scan 1 (A), susceptibility phantom in scan 2 (acquired 4 months after scan 1) (B) and sphere phantom in scan 2 (C). Quantitative results for entire volumes are shown in Tables 2 and 3. 
were used to compute the first-order phase correction terms without the effect of the field inhomogeneity of the sample.

\section{Results}

Table 1 shows that $\alpha$ was consistently estimated to be -0.10 radians for all the phantom data from the same scanner. The value of $\beta$ estimated with Eq. (8), however, changes for different scans. A surface plot of the cost function in the range $-1.0 \leq \alpha \leq 1.0$ and $-4.0 \leq \beta \leq 4.0$ was computed to verify that the estimated values correspond to global minimum points. Fig. 4 shows the true, dual-echo and corrected dual-echo field map profiles of a single column of the sphere phantom in the readout direction. The first-order phase error in Fig. 4B is corrected as observed in Fig. 4C. The third row of Table 1 shows the respective values of $\beta$, computed using the empirical method. It is noted that they closely approximate the $\beta$ values computed with Eq. (8) shown in the second row of Table 1. The values of $\alpha$ and $\beta$ (nonempirical) in Table 1 were then used to correct the respective dual-echo field maps of phantoms in each scan session.

Fig. 5(A-C) show sample slices from the susceptibility phantom from scan time 1 (A) and scan time 2 (B) and sphere phantom from scan time 2 (C). In each subfigure, the off-resonance maps are shown in rows of sample slices selected from the dual-echo data without correction (top), after applying the affine phase correction terms (middle) and two separate single-echo acquisitions (bottom). It is evident that massive phase wrapping in the corrected DEFGRE offresonance maps in the middle row due to the first-order phase shift has been removed. The root mean square error (RMSE) values between the dual-echo and corrected dualecho field maps, and the ground truth field maps over all 68 slices in each phantom scan are shown in Table 2. The RMSE values (ranging from 0.17 to $0.43 \mathrm{ppm}$ ) for the corrected dual-echo off-resonance maps were relatively low for field map estimation with the dual-echo pulse sequence in Fig. 2.

Results from the three human subject scans confirm that the same value of $\alpha$ obtained in Table 1 gives good correction results for the same scanner over a period of 2 years. Prior to obtaining empirical approximations of $\beta$, the mean off-resonance value of homogeneous regions of

Table 2

Off-resonance RMSE values in hertz and parts per million $\left(B_{0}=1.5 \mathrm{~T}\right)$ between each phantom's corrected dual-echo field map (using parameters computed in Table 1) and corresponding field maps computed with the standard field map method (using 2D SPGR data)

\begin{tabular}{|c|c|c|}
\hline \multicolumn{3}{|l|}{ RMSE (Hz, ppm) } \\
\hline Scan 1 (susceptibility) & $\begin{array}{l}\text { Scan } 2 \text { (4 months later) } \\
\text { (susceptibility) }\end{array}$ & $\begin{array}{l}\text { Scan } 2 \text { (4 months } \\
\text { later) (sphere) }\end{array}$ \\
\hline $27.26 \mathrm{~Hz}, 0.43 \mathrm{ppm}$ & $23.43 \mathrm{~Hz}, 0.37 \mathrm{ppm}$ & $11.16 \mathrm{~Hz}, 0.17 \mathrm{ppm}$ \\
\hline
\end{tabular}

Table 3

Off-resonance RMSE values in hertz and parts per million $\left(B_{0}=1.5 \mathrm{~T}\right)$ between each human subject's corrected dual-echo field map (using $\alpha=-0.10$ with $\beta$ computed empirically for each scan) and corresponding field maps computed with the standard field map method (using 3D SPGR data)

RMSE (Hz, ppm)

\begin{tabular}{lll}
\hline Subject 1 & Subject 2 & Subject 3 \\
\hline $33.88 \mathrm{~Hz}, 0.53 \mathrm{ppm}$ & $27.98 \mathrm{~Hz}, 0.44 \mathrm{ppm}$ & $32.03 \mathrm{~Hz}, 0.50 \mathrm{ppm}$ \\
\hline
\end{tabular}

Only pixels with intensity values above $10 \%$ of the maximum image intensity of the respective data sets are used in the computation of the RMSE values.

three human subject brains over 10 slices each, $f_{\text {sep,mean, was }}$ computed to be $18.89 \mathrm{~Hz}$. The DEFGRE off-resonance map for each subject was corrected with the first-order phase term $\alpha$, and the mean off-resonance values, $f_{\text {dual,mean, }, 1}$, $f_{\text {dual,mean, } 2}$ and $f_{\text {dual,mean, } 3}$, of corresponding homogeneous regions over 10 slices of the resultant data were computed to be $165.54,-130.30$ and $188.84 \mathrm{~Hz}$, respectively. The corresponding value of $\beta$ (radians) for the $i^{\text {th }}$ subject is obtained by $\beta_{\mathrm{i}}=2 \pi\left(f_{\text {sep,mean }}-f_{\text {dual,mean }, \mathrm{i}}\right) \Delta T E_{\text {dual }}$, which yield $\beta_{1}=-2.40 \mathrm{rad}, \beta_{2}=2.44 \mathrm{rad}$ and $\beta_{3}=-2.78 \mathrm{rad}$ for the three subjects, respectively. Table 3 shows that the RMSE values for the corrected DEFGRE using the empirically determined values of $\beta$, compared to the reference 3D SPGR off-resonance maps, are relatively low (ranging from 0.44 to $0.53 \mathrm{ppm}$ ), indicating that the corrected offresonance maps are close to the 3D SPGR off-resonance maps. This RMSE comparison is performed to determine if the empirically computed values of $\beta$ have corrected most of the zero-order phase shift. Only pixels having significant MR signal (i.e., image intensity values above $10 \%$ of the maximum image intensity value) were used in computing the RMSE. Fig. 6 shows DEFGRE offresonance map slices for three of the subjects before and after the affine phase correction with empirically determined values of $\beta$. It is observed that the zero- and first-order phase errors have been largely removed after the phase correction procedure.

\section{Discussion}

The first-order phase correction term $\alpha$ was computed with field maps generated from phantom data acquired with a single-echo SPGR at two different echo times and a dualecho sequence and was used to remove the linearly varying phase error in field maps acquired using the same dual-echo protocol on the same scanner. The correction was tested on multiple sets of human brain data as well as phantom data that exhibit susceptibility artifacts. The results show that $\alpha$ was observed to be constant on the same scanner over a period of 2 years. The computation process is straightforward, and no elaborate phase unwrapping is required to correct for the first-order component of the phase error. The estimation of $\alpha$ on each scanner needs to be done only once and can be used to perform field map estimation with dual- 
A
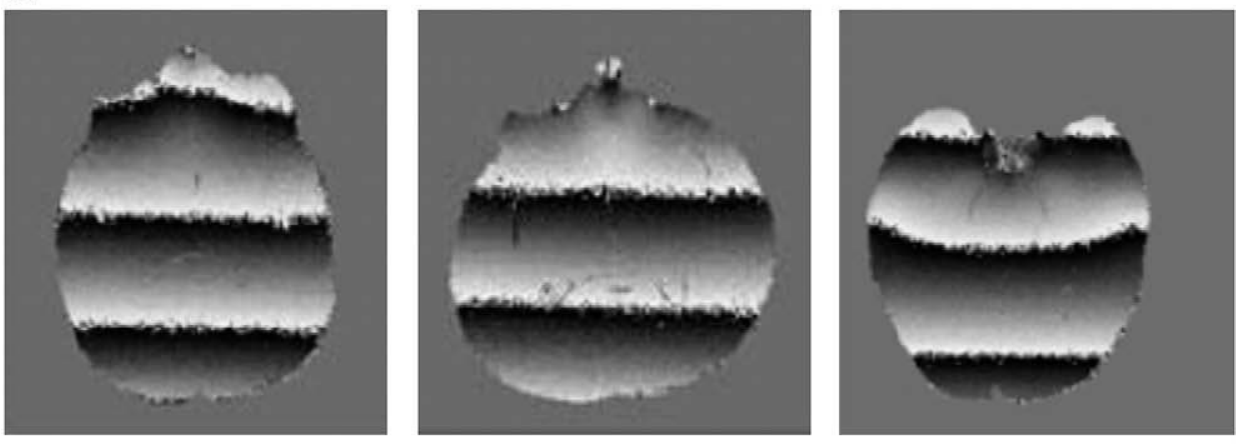

B
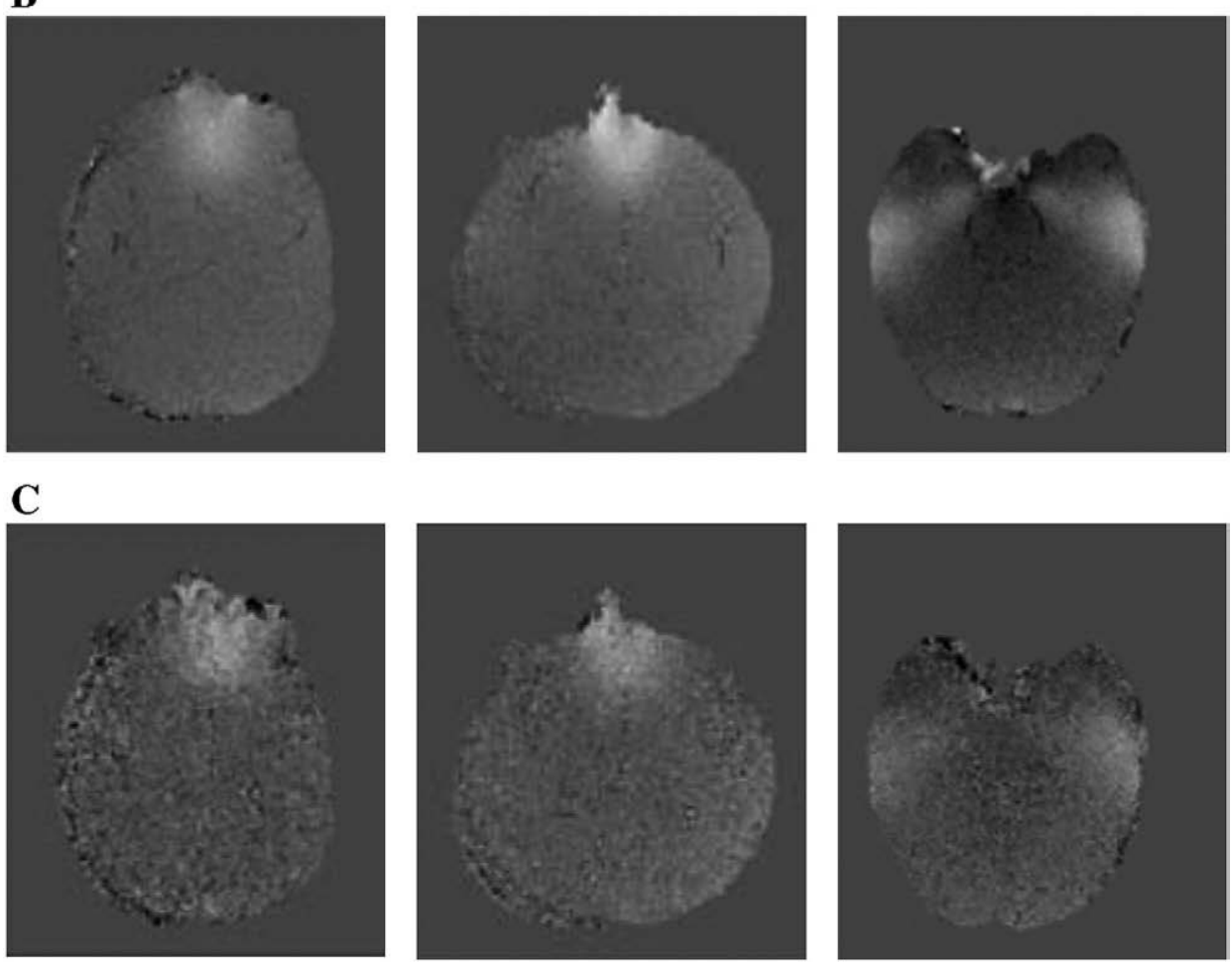

Fig. 6. Subject 1 (first column), subject 2 (second column) and subject 3 (third column) off-resonance slices from uncorrected DEFGRE data [direct application of Eq. (7)] (A), DEFGRE field map corrected with affine phase term (empirically determined $\beta$ ) (B) and standard two single-echo 3D SPGR data (C). Note that the linearly varying phase error in (A) has been removed in (B). Part (A) is displayed on a scale from -1500 to $1500 \mathrm{~Hz}$, while (B) and (C) are both displayed on a scale from $-100 \mathrm{~Hz}$ to $200 \mathrm{~Hz}$.

echo data thereafter. In the event that the scanning environment changes, the recalibration can be done by following the simple protocol set up to acquire data with the two single-echo GRE and DEFGRE sequences using a homogeneous spherical phantom.

Although the zero-order phase term $\beta$ varies with different scan sessions, we have proposed an empirical method to approximate it using only DEFGRE data and an average off-resonance value computed from suitable homogeneous regions of objects previously scanned with the two single-echo SPGR protocols on the same scanner. This empirical method yielded corrected DEFGRE off-resonance maps that had relatively low off-resonance RMSE values ( $\leq 0.53 \mathrm{ppm}$ for human subjects at $B_{0}=1.5 \mathrm{~T}$ ). As stated previously, the off-resonance maps computed with the two single-echo acquisitions are prone to motion artifacts since the data are acquired from two separate echoes. Thus, the RMSE values for the human subject data are meant to be approximations of how close the corrected DEFGRE offresonance maps are to the standard off-resonance maps, but not measures of accuracy in the corrected DEFGRE field maps. This is useful information only because in the absence of a ground truth field map without motion, it shows that the corrected DEFGRE estimates do not deviate significantly from the standard field map. For the phantom data, the field maps computed using the standard method is a closer approximation to the ground truth field map since the phantoms do not move during the scans. Thus, using the standard field maps as ground truth field maps, the RMSE values of phantom dual-echo data after phase shift 
correction are better measures of the accuracy of the corrected DEFGRE method.

Other factors like the different field map SNR values obtained with different $\triangle T E$ values used in the 3D SPGR and DEFGRE protocols may influence the accuracy of the RMSE values. It is observed that the field maps computed using the two separate single-echo acquisition methods are noisier than the corrected DEFGRE field maps. This is true for both phantom and human data. For the phantom experiments, the standard deviation values of homogeneous field map regions, which approximate the field map noise levels, were 4.9, 6.0 and $6.9 \mathrm{~Hz}$ for single-echo, while the values for the corresponding regions in the respective dual echo data sets were $3.15,0.4$ and $4.3 \mathrm{~Hz}$. Similarly, the human field map measurements were 20.6, 24.2 and $19.7 \mathrm{~Hz}$ for single-echo and 15.6, 12.5 and $11.6 \mathrm{~Hz}$ for dual-echo experiments. Among other reasons, this phenomenon may be due to $\Delta \mathrm{TE}_{\text {dual }}>\Delta \mathrm{TE}_{\mathrm{sep}}$, combined with motion-induced noise. The human subjects' noise standard deviation values are larger than phantom noise standard deviation values, which may be attributed to additional noise sources in humans, i.e., motion, body thermal noise, etc. Performing a future field map SNR study of phantom (with and without motion) and human data with $\Delta \mathrm{TE}_{\text {dual }}=\Delta \mathrm{TE}_{\text {sep }}$ may aid in quantifying any motion-related SNR gain in using the DEFGRE field map estimation method compared to the standard field map method.

It is ideal to keep the $\Delta \mathrm{TE}$ values equal in order to be able to compare SNRs in field maps fairly. For field map measurements, $\Delta$ TEs were kept as close as possible for the standard and dual-echo sequences while avoiding spontaneous changes in other acquisition parameters due the scanner's built-in timing restrictions in choosing TEs. Our key motive was to use the DEFGRE sequence for field map estimation as it was with the limitations in sequence timing included, and results strongly suggest that the affine phase error model holds over time ( 2 years).

The computation of $\alpha$ and $\beta$ in the initial calibration stage to minimize Eq. (8) was done via the Nelder-Mead simplex algorithm. The cost function is periodic with respect to $\beta$ and has many local minimum points with respect to $\alpha$ in the vicinity of the global minimum of Eq. (8). This may cause the Nelder-Mead algorithm to yield a local minimum point as the optimum solution. An alternative optimization method is to perform a line search with respect to $\alpha$ and use the solution of $\alpha$ in a derived maximum likelihood analytical solution for $\beta$ assuming a white Gaussian observation model, as shown in Appendix A.

\section{Conclusions}

The dual-echo bipolar readout gradient technique offers an efficient way of collecting data and computing static field maps with reduced motion-induced errors compared to the widely used two separate single-echo acquisition methods. The affine parameters modeling the phase error inherent in the dual-echo bipolar readout gradient technique is estimated with data from a phantom of homogeneous medium where the field inhomogeneity is mainly system-induced. The estimated phase correction parameters are then applied to DEFGRE data of an air-tissue susceptibility phantom. Results have shown that the first-order phase error term stays constant with time as expected with the same scanner using the same DEFGRE protocol parameters, allowing the technique to be used for human subject field map estimation once the first-order phase error term has been characterized. The first-order term is due to the readout imbalance, which is scanner-dependent, and yields similar $k$-space shifts in each readout line acquired with the dual-echo acquisition. The zeroth-order term has off-resonance contributions from other sources such as heating effects of coils, depending on the object being scanned. The phase error from the zerothorder term changes with different scan sessions but can be estimated empirically using the previously scanned two single-echo field maps. The proposed method has been tested on three human subjects, and the results strongly suggest that the DEFGRE pulse sequence can yield good field map estimates. The relatively low RMSE values (ranging from 0.17 to $0.43 \mathrm{ppm}$ ) for the corrected dualecho off-resonance maps at $1.5 \mathrm{~T}$ suggest that the affine phase error model is suitable for field map estimation with the dual-echo pulse sequence in Fig. 2. Since the first-order correction term depends largely on how the readout gradient switches and not on how strong the $B_{0}$ field is, an affine phase error model is expected to hold for images from different field strengths. The value of the linear phase term may be different for different scanners but should be constant for any one scanner. Future work includes an evaluation of the proposed field map estimation method at $3.0 \mathrm{~T}$ and the investigation of methods to improve the reliability of the zero-order phase error estimate.

\section{Appendix A. Maximum likelihood estimator for $\beta$}

As an alternative to using direct search methods to compute the phase correction terms $\alpha$ and $\beta$, a maximum likelihood estimator can be derived for $\beta$ while $\alpha$ can be estimated via a line search. Let the general observation model be

$Z=m e^{j \theta}+N$,

where $m$ is an unknown magnitude, $\theta$ is the unknown phase of interest and $N$ is complex, zero-mean Gaussian noise with variance $\sigma^{2}$, i.e.,

$\left[\begin{array}{l}\operatorname{real}(N) \\ \operatorname{imag}(N)\end{array}\right] \sim N\left(\left[\begin{array}{l}0 \\ 0\end{array}\right],\left[\begin{array}{ll}\sigma^{2} & 0 \\ 0 & \sigma^{2}\end{array}\right]\right)=N\left(\mu_{N}, \sum_{N}\right)$. Thus, the likelihood function can be written as follows:

$f_{\mathrm{z}}(z ; \theta)=\frac{1}{\sqrt{(2 \pi)^{n}\left|\sum_{N}\right|}} e^{-\left(z-\mu_{N}\right)^{H} \sum_{N}^{-1}\left(z-\mu_{N}\right) / 2}$,

where $\mu_{N}=E[Z]$. Taking the logarithm of Eq. (A.2) and 
removing the terms that are independent of $\theta$, the $\log$ likelihood can be written as

$$
\begin{aligned}
\log f_{\mathrm{z}}(z ; \theta) & \equiv-\frac{1}{2}\left|z-m e^{j \theta}\right|^{2} \\
& =-\frac{1}{2}\left(z-m e^{j \theta}\right)\left(z^{*}-m e^{-j \theta}\right) \\
& \equiv \frac{1}{2} z m e^{-j \theta}+\frac{1}{2} z^{*} m e^{j \theta} \\
& =m \operatorname{Re}\left\{z^{*} m e^{j \theta}\right\} \\
& =m|z| \cos (\theta-\angle z)
\end{aligned}
$$

where " $\equiv "$ denotes equality after removing terms that are independent of $\theta$. For the dual-echo field map estimation problem, let

$$
\begin{aligned}
z & =I_{\text {dual }}(r) I_{\text {sep }}^{*}(r) /\left|I_{\text {sep }}(r)\right| \\
& =\left|M_{\text {dual }}(r)\right| e^{j\left\{\angle I_{\text {dual }}(r)-\Delta \hat{\omega}_{\text {sep }}(r) \Delta T E_{\text {sep }}\right\}}+\varepsilon(r)
\end{aligned}
$$

Since the true magnitude of $I_{\text {dual }}$ is approximately equal to the observed noisy magnitude of $z$, we assume $m \approx|z|$. The magnitude and angle of $z$ can be stated as $|z| \approx\left|M_{\text {dual }}(r)\right|$ and $\angle z \approx \angle I_{\text {dual }}(r)-\Delta \hat{\omega}_{\text {sep }}(r) \Delta T E_{\text {sep. }}$. In reality, $\left|M_{\text {dual }}(r)\right|$ is also unknown and is approximated by $\left|I_{\text {dual }}(r)\right|$. By having $\theta=\alpha x+\beta$ where $x$ is the frequency encoded readout direction, Eq. (A.3) can be written as follows:

$$
\begin{aligned}
& \log f_{\mathrm{z}}(z ; \theta) \\
& \propto|z|^{2} \cos (\angle z-\theta) \\
& =\left|M_{\text {dual }}\right|(x)^{2} \cos \left(\angle I_{\text {dual }}(x)-\Delta \hat{\omega}_{\text {sep }}(x) \Delta T E_{\text {sep }}-\alpha x-\beta\right) \\
& =\left|M_{\text {dual }}(x)\right|^{2} \cos (\varphi(x)-\beta)
\end{aligned}
$$

where $\varphi(x)=\angle I_{\text {dual }}(x)-\Delta \hat{\omega}_{\text {sep }}(x) \Delta \mathrm{TE}_{\text {sep }}-\alpha x$.

Using the identity $\cos (\varphi(x)-\beta)=\cos \beta \cos \varphi(x)+\sin \beta \sin$ $\varphi(x)$, Eq. (A.5) can be expressed as

$$
\begin{aligned}
\psi(x, \alpha, \beta)= & \sum_{x=1}^{N}\left|M_{\text {dual }}(x)\right|^{2} \cos (\varphi(x)-\beta) \\
= & {\left[\sum_{x=1}^{N}\left|M_{\text {dual }}(x)\right|^{2} \cos \varphi(x) \cos (\beta)\right] } \\
& +\left[\sum_{x=1}^{N}\left|M_{\text {dual }}(x)\right|^{2} \sin \varphi(x) \sin \beta\right]
\end{aligned}
$$

where $N$ is the number of pixels used in a readout line. Assuming $\alpha$ can be found via a line search, the maximum likelihood estimator of $\beta$ can be obtained by taking the derivative of Eq. (A.6) with respect to $\beta$, as follows:

$$
\begin{aligned}
& \frac{\partial \psi(x, \beta)}{\partial \beta}=-\left[\sum_{x=1}^{N}\left|M_{\text {dual }}(x)\right|^{2} \cos \varphi(x) \sin (\beta)\right] \\
&+\left[\sum_{x=1}^{N}\left|M_{\text {dual }}(x)\right|^{2} \sin \varphi(x) \cos \beta\right]=0 \\
& \Rightarrow \beta=\arctan \left[\frac{\sum_{x=1}^{N}\left|M_{\text {dual }}(x)\right|^{2} \sin \varphi(x)}{\sum_{x=1}^{N}\left|M_{\text {dual }}(x)\right|^{2} \cos \varphi(x)}\right]
\end{aligned}
$$

In summary, the maximum likelihood estimator for $\beta$ in Eq. (A.7) can be substituted into Eq. (A.5) and a line search performed with respect to $\alpha$ to maximize Eq. (A.5). The resultant solution for $\alpha$ is then substituted into Eq. (A.7) to yield a solution for $\beta$.

\section{References}

[1] Jezzard P, Balaban RS. Correction for geometric distortion in echo planar images from B0 field variations. Magn Reson Med 1995;34: $65-73$.

[2] Cusack R, Brett M, Osswald K. An evaluation of the use of magnetic field-maps to undistort echo-planar images. Neuroimage 2003;18: $127-42$.

[3] Schneider E, Glover G. Rapid in vivo proton shimming. Magn Reson Med 1991;18:335-47.

[4] Park H, Kim Y, Cho Z. Fast gradient-echo chemical-shift imaging. Magn Reson Med 1988;7:340-5.

[5] Webb P, Macovski A. Rapid, fully automatic, arbitrary-volume in vivo shimming. Magn Reson Med 1991;20:113-22.

[6] Kim B, Boes JL, Bland PH, Chenevert TL, Meyer CR. Motion correction in fMRI via registration of individual slices into an anatomical volume. Magn Reson Med 1999;41:964-72.

[7] Wirestam R, Salford LG, Thomsen C, Brockstedt S, Persson BRR, Stahlberg F. Quantification of low-velocity motion using a navigatorecho supported MR velocity-mapping technique: application to intracranial dynamics in volunteers and patients with brain tumours. Magn Reson Imaging 1997;15(1):1-11.

[8] Roopchansingh V, Cox RW, Jesmanowicz A, Ward BD, Hyde JS. Single-shot magnetic field mapping embedded in echo-planar timecourse imaging. Magn Reson Med 2003;50:839-43.

[9] Reber PJ, Wong EC, Buxton RB, Frank LR. Correction of offresonance-related distortion in echo-planar imaging using EPI-based field maps. Magn Reson Med 1998;39:328-30.

[10] Sutton BP, Noll DC, Fessler JA. Fast, iterative image reconstruction for MRI in the presence of field inhomogeneities. IEEE Trans Med Imaging 2003;22:178-88.

[11] Munger P, Crelier GR, Peters TM, Pike GB. An inverse problem approach to the correction of distortion in EPI images. IEEE Trans Med Imaging 2000;19:681-9.

[12] Ghiglia DC, Pritt MD. Two-dimensional phase unwrapping: theory, algorithms, and software. Wiley: New York; 1998.

[13] Cusack R, Papadakis N. New robust 3-D phase unwrapping algorithms: application to magnetic field mapping and undistorting echoplanar images. Neuroimage 2002;16:754-64. 